\title{
ON THE PASSAGE TO EQUILIBRIUM OF GIBBSIAN ENSEMBLES
}

\author{
BY \\ CLIFFORD C. BROWN
}

Introduction. The following problem was considered by Gibbs [1]. Although his comments on it were intuitive in nature, they contain what appears to be the essential idea behind the solution. The problem goes as follows: Given an ensemble of mechanical systems, each of which is an ideal gas in a box, with all the boxes alike, we can define the ensemble entropy $S$ by

$$
S=-k \int \rho \log \rho d V
$$

where $k$ is Boltzmann's constant, $\rho$, the system density in configuration space, and $V$ the $2 n$-dimensional Lebesgue measure in the $2 n$-dimensional configuration space. Gibbs proved that $S$ so defined has properties which, for certain functions $\rho$, are analogous to those of thermodynamical entropy. If, however, we can expect $S$ to behave like thermodynamical entropy, then if, at time $t=t_{0}$, the containers belonging to the gas systems in the aforementioned ensemble are all suddenly and simultaneously doubled in volume (e.g. by removal of partitions, etc.), the quantity $S$ should, in the period $t>t_{0}$, approach a value that is greater than its value at time $t=t_{0}$. Such a change in the value of $S$ appears to be impossible since, by Liouville's theorem, $S$ as defined above is a constant of motion for the ensemble.

Gibbs removed this difficulty in a way which is most easily understood by examining a special case. Let each of the systems in the ensemble be, instead of an ideal gas, a single degree of freedom rigid rotator. Configuration space now has two dimensions, $q$ and $p$, the angular displacement and momentum respectively. We adopt the convention that $\pi \geqq q>-\pi$. We suppose that at time $t=0$ the system density is given by

$$
\rho_{0}(p, q)=\left\{\begin{array}{cl}
f(p) & \text { when } 0 \geqq q>-\pi, \\
0 & \text { when } \pi \geqq q>0
\end{array}\right.
$$

where $f(p)$ is some measurable function of $p$ alone. The item of major interest will be the manner in which the region $0 \geqq q>-\pi$ maps, as time proceeds, into the region $\pi \geqq q>-\pi$ as a result of the transformation specified by the laws of mechanics. The various images of this region for various times $t=0,1,2,4,8,16$ are shown in Figure 1. It is clear from Figure 1 how the ensemble of systems under consideration approaches ensemble equilibrium. It is even possible to write down a final "equilibrium density,"

Received by the editors December, 1958; and, in revised form, November 30, 1959. 

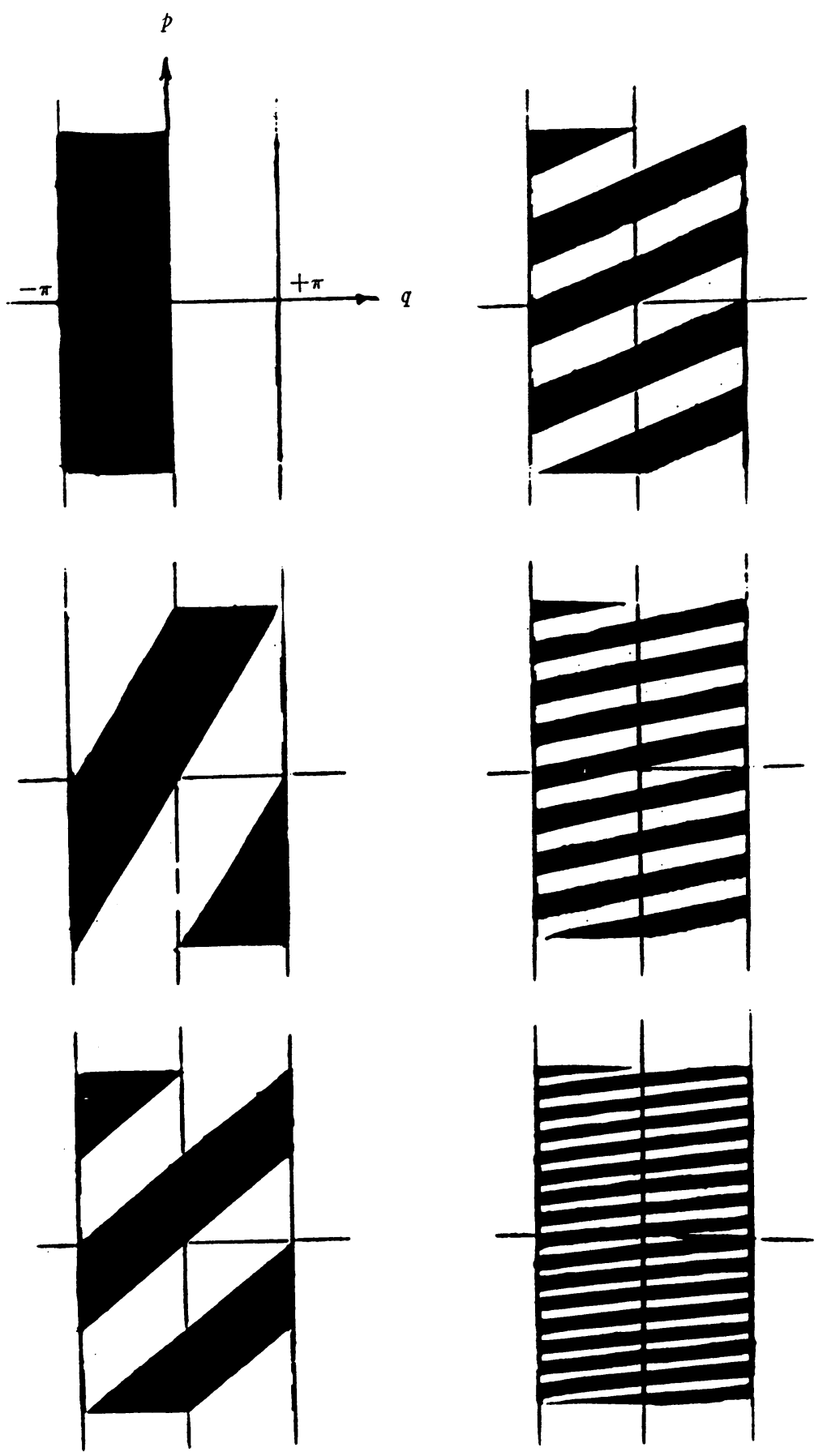

Figure 1. 


$$
\rho_{\infty}(p, q)=f(p) / 2 \quad \text { for } \pi \geqq q>-\pi .
$$

A precise definition of equilibrium density will be developed in this paper.

The usual procedure for dealing mathematically with this aspect of statistical mechanics [2] is to work not with the actual density function as it develops through time from $\rho_{0}(p, q)$, but with the "coarse grained density" defined by

$$
P(p, q)=\frac{1}{V(E)} \int_{E} \rho d V
$$

where $E$ is a cube of given nonzero volume $V(E)$ and with center at $(p, q)$. In reference [2] an argument is given to show that the quantity $\bar{S}$, where

$$
\bar{S}=-k \int P \log P d V
$$

cannot decrease in time during passage to equilibrium. Considering the special case above, it is clear that it will sometimes increase.

The procedure of defining a coarse grained density and stating important theorems in terms of it seems unnatural. This paper deals with the passage to equilibrium in a rigorous manner, and avoids any reliance on the coarse grained density concept. Unfortunately, there remain important outstanding questions whose answers must wait for far deeper understanding of the equations of mechanics than has been achieved up to the present time.

It will be apparent from this treatment that the mathematical expression of the approach to equilibrium is very similar to what is normally referred to as "weak convergence" by mathematicians. This is in partial agreement with a conjecture by $\mathrm{H}$. Grad [3]. It has been so far impossible to establish reasonable conditions which assure weak convergence in the strictest sense. Heavier reliance on the laws of mechanics seems to be necessary if such a thing is to be proved.

1. Definition of the measure space. Let $M$ be the set of all possible static positions of a given $n$-degree of freedom mechanical system. We assume $M$ to be an $n$ dimensional, $C^{\infty}$, Lindelöf manifold in which the coordinate values over a given coordinate neighborhood are denoted by $q_{1}, \cdots, q_{n}$.

Let $F_{q}$ be the dual space to the tangent space at $q \in M$. Let $B=U_{q \in M} F_{q}$ be the corresponding vector bundle over $M$. We define the "elementary canonical coordinate systems" in $B$ as follows. Let $(\mathcal{U}, \phi)$ be a coordinate system in $M$. For $q \in \mathcal{U}$, with $\phi(q)=\left(q_{1}, \cdots, q_{n}\right) \in R^{n}$, let $X$ be in $F_{q}$. Then $X$ has the coordinates $\left(q_{1}, \cdots, q_{n} ; p_{1}, \cdots, p_{n}\right) \in R^{2 n}$, where

$$
p_{i}=X\left(\frac{\partial}{\partial q_{i}}\right), \quad(i=1, \cdots, n)
$$


where $\partial / \partial q_{i}$ is the natural basis for the tangent space at $q$ in the $(\mathcal{u}, \phi)$ coordinate system. The coordinate neighborhoods in $B$ are, by the definition of a vector bundle, the sets of the form $\mathcal{U}=\cup_{q \in \mathcal{U}} F_{q}$, where $\mathcal{U}$ is a coordinate neighborhood in $M$.

DEFinition. Let $(v, \eta)$ be an elementary canonical coordinate system in $B$. Let $X \in \mathcal{V}$. Let $f$ and $g$ be differentiable functions defined on $v$. We define the "Poisson bracket," $[f, g]$ of $f$ and $g$ with respect to $(v, \eta)$ at $X$ by

$$
[f, g]=\sum_{i=1}^{n}\left(\frac{\partial f}{\partial q_{i}} \frac{\partial g}{\partial p_{i}}-\frac{\partial f}{\partial p_{i}} \frac{\partial g}{\partial q_{i}}\right) \text {. }
$$

where the $q_{i}, p_{i}$ are the coordinate values in the $(\vartheta, \eta)$ system, and where the partial derivatives are evaluated at $X$.

REMARK. Let $\left(v_{1}, \eta_{1}\right)$ and $\left(v_{2}, \eta_{2}\right)$ be two elementary canonical coordinate systems in $B$, with $v_{1} \cap v_{2}$ nonempty. Let the coordinate values in the $\left(v_{1}, \eta_{1}\right)$ system be $\left(q_{11}, \cdots, q_{1 n} ; p_{11}, \cdots, p_{1 n}\right)$. Then the Poisson brackets [ , ] of the $q_{1 i}, p_{1 j}$ with respect to $\left(v_{2}, \eta_{2}\right)$ at $X \in v_{1} \cap v_{2}$ satisfy the relations,

$$
\left[q_{1 i}, q_{1 j}\right]=0, \quad\left[q_{1 i}, p_{1 j}\right]=\delta_{i j}, \quad\left[p_{1 i}, p_{1 j}\right]=0 .
$$

It is well known that these relations imply that $[f, g]$ with respect to $\left(v_{1}, \eta_{1}\right)$ at $X \in v_{1} \cap v_{2}$ is equal to $[f, g]$ with respect to $\left(v_{2}, \eta_{2}\right)$ at $X$. We can therefore define a more general coordinate system in $B$.

Definition. Given a coordinate system in $B,(W, \mu)$, let its $2 n$ coordinate values be denoted by $\left(Q_{1}, \cdots, Q_{n} ; P_{1}, \cdots, P_{n}\right)$. We call $(W, \mu)$ a canonical coordinate system in $B$ if, for any elementary canonical coordinate system $(v, \eta)$ with $w \cap v$ nonempty, $\mu \circ \eta^{-1}$ is $C^{\infty}$ in $\eta(v \cap W)$, and the Poisson brackets of the $Q_{i}, P_{j}$ with respect to the $(v, \eta)$ system at $X \in V \cap w$ satisfy the relations

$$
\left[Q_{i}, Q_{j}\right]=0, \quad\left[Q_{i}, P_{j}\right]=\delta_{i j}, \quad\left[P_{i}, P_{j}\right]=0 .
$$

It is clear from this definition that the Poisson bracket of two functions $f$ and $g$ has the same value in any canonical coordinate system.

From their construction, the canonical coordinate systems cover $B$.

A theorem due to Poincaré states that if $\left(W_{1}, \mu_{1}\right)$ and $\left(W_{2}, \mu_{2}\right)$ are two canonical coordinate systems in $B$ with $W_{1} \cap W_{2}$ nonempty, then the square of the determinant of the transformation $\mu_{1} \circ \mu_{2}^{-1}$ from $\mu_{2}\left(w_{1} \cap w_{2}\right)$ to $\mu_{1}\left(w_{1} \cap w_{2}\right)$ is unity. This follows directly from the above definition of canonical coordinates. This enables us to define a measure on $B$ in a simple way. Let $E \subset B$ be contained entirely in some canonical coondinate neighborhood $w$, belonging to the system $(W, \mu)$. We will say that $E$ is measurable if $\mu(E) C R^{2 n}$ is Borel measurable in $R^{2 n}$. The Lindelöf property of $M$ allows the extension of this definition to any set in $B$. If $E \subset$ w, its measure is the Borel measure of $\mu(E)$. The corresponding measure, definable through the Lindelöf 
property of $M$, of any measurable set in $B$ will be denoted by $V$, and called the Borel measure over $B$.

2. Further definitions and assumptions.

Definition. Let $X \in B$. There is exactly one $q \in M$ such that $X \in F_{q}$. We denote by $-X$ that element $Y \in F_{q}$ for which $X+Y=0$.

Definition. Let $E \subset B$. We denote by $E^{*}$ that set in $B$ for which $X \in E$ if and only if $-X \in E^{*}$.

Definition. Let $E_{1}$ and $E_{2}$ be measurable subsets of $B$. We define

$$
E_{1} \Delta E_{2} \equiv\left(E_{1}-E_{2}\right) \cup\left(E_{2}-E_{1}\right) \text {. }
$$

The quantity $V\left(E_{1} \Delta E_{2}\right)$ has important properties which are discussed in [5, Chapter VIII]. Two trivial ones, useful for proving some remarks to follow, are

$$
\begin{aligned}
& V\left(E_{1} \Delta E_{2}\right)=V\left(E_{1} \cup E_{2}\right)-V\left(E_{1} \cap E_{2}\right), \\
& V\left(E_{1} \Delta E_{2}\right)=V\left(E_{1}-E_{2}\right)+V\left(E_{2}-E_{1}\right) .
\end{aligned}
$$

Let $\Omega=-\Omega$ be a measurable set in $B$. For real $t \geqq 0$, let $f_{t}$ be a mapping of $\Omega$ into $\Omega$. We impose the following conditions on $f_{t}$.

(A) $E$ is measurable if and only if $f_{t}^{-1}(E)$ is. $E$ is measurable if and only if $f_{t}(E)$ is.

(B) $V(E)=V\left(f_{t}(E)\right)$.

(C) Let $s \geqq 0, t \geqq 0$. Then for all measurable $E \subset \Omega, f_{t+s}(E)=f_{t}\left(f_{s}(E)\right)$.

(D) There is an increasing sequence $\left\{F_{n}\right\}$ of measurable sets, each of finite $V\left(F_{n}\right)$, such that $\bigcup_{n} F_{n}=\Omega$ and, for all $t \geqq 0, V\left(F_{n} \Delta f_{t}\left(F_{n}\right)\right)=0$.

Condition (D) is obviously true for systems whose Hamiltonians are such that the set $\{X:|H(X)|<n\}$ is of finite measure for each $n$. There are many systems of this type. There are also important cases, however, where such sets are not of finite measure. In the case of a particle moving in a spherical gravitational field, for example, the set $\{X: \alpha<H(X)<\beta\}$ is not of finite measure for any $\alpha<\beta$. If we choose for $\Omega$ the set $\{H<0\}$, corresponding to bound orbits, the existence of a sequence $\left\{F_{n}\right\}$ can be proved.

We now make the following remarks without proof. They make possible the approximate extension of conditions (A) to (D) to all values of $s$ and $t$.

REMARK (1). Conditions (A) and (D) imply that $V\left(\Omega \Delta f_{t}(\Omega)\right)=0$ for all $t \geqq 0$.

REMARK (2). Condition (A) and Remark (1) imply that $V\left(E \Delta f_{t}\left(f_{t}^{-1}(E)\right)\right)$ $=0$ for all $t \geqq 0$.

REMARK (3). Remark (2) and Conditions (A) and (B) imply that $V(E)$ $=V\left(f_{t}^{-1}(E)\right)$.

REMARK (4). Conditions (A) and (B) imply that $V(E \Delta F)=0$ if $V\left(f_{t}(E) \Delta f_{t}(F)\right)=0$.

REMARK (5). Remark (4) and Condition (A) imply that $V\left(E \Delta f_{t}^{-1}\left(f_{t}(E)\right)\right)$ $=0$. 
Remark (6). Remark (5) and Conditions (A) and (B) imply that

$$
\begin{aligned}
V\left(f_{t}(E) \Delta f_{t}(F)\right) & =V(E \Delta F), \\
V\left(f_{t}(E) \cap f_{t}(F)\right) & =V(E \cap F) .
\end{aligned}
$$

Remark (7). Remark (6) and Condition (C) imply that $V\left(f_{0}(E) \Delta E\right)=0$.

Remark (8). Define $f_{-t}(E)=f_{t}^{-1}(E)$ for $t>0$. Then Condition (C) and Remarks (2), (3), (5), (6) and (7) imply that $V\left(f_{t}\left(f_{t}(E)\right) \Delta f_{t+t}(E)\right)=0$ for all real $s$ and $t$.

Remark (9). Condition (D), Remark (3) and Remark (5) imply that $V\left(F_{n} \Delta f_{t}\left(F_{n}\right)\right)=0$ for all $t$.

In these remarks, it is to be understood that $E$ and $F$ are Borel measurable subsets of $\Omega$. We now append one further condition to Conditions (A)-(D).

Condition (E). $V\left(f_{t}\left(E^{*}\right) \Delta\left(f_{-t}(E)\right)^{*}\right)=0$ for all $t$ and measurable $E$.

This condition is a form of what is commonly referred to in classical mechanics as the principle of reversibility of time. The condition as it is stated here is a consequence of the symmetry of the Hamiltonian under change of sign of the momentum coordinates in any elementary canonical coordinate system.

Definition. We will use the notation, $f_{t}(E)=E_{t}$.

Given a class $\left\{f_{t}\right\}$ of mappings of $\Omega$ into $\Omega$ satisfying conditions (A) through (E) above, we can, from a given totally $\sigma$-finite absolutely continuous measure $I$ over $\Omega$ define a class of measures $\left\{I_{t}\right\}$, which are also totally $\sigma$-finite and absolutely continuous, by

$$
I_{t}(E)=I\left(E_{-t}\right) .
$$

This definition is equivalent to

$$
I_{t}\left(E_{t}\right)=I(E)
$$

and for this reason, we call $\left\{I_{t}\right\}$ the conservative class of measures generated by $I$.

REMARK (10). There exists a measurable function $\rho \geqq 0$ defined on $\Omega$ with the property

$$
I_{t}(E)=\int_{E_{-t}} \rho d V,
$$

for all measurable $E$. The function $\rho$ is the Radon-Nikodym derivative of $I$ with respect to $V$.

REMaRK (11). Condition (B) and the absolute continuity of $I$ imply that if $I$ is totally finite, $\left\{I_{t}\right\}$ is a uniformly absolutely continuous class of totally finite measures.

3. Theorems on equilibrium. The following pair of lemmas from measure theory will be found useful in what follows. They will not be proved in this paper. 
LemmA (A). Let $\nu$ be a totally $\sigma$-finite measure. Let $\left\{M_{t}\right\}$ be a class of totally finite measures which is uniformly absolutely continuous and such that, for some totally finite absolutely continuous measure $M(E), \lim _{t \rightarrow \infty} M_{t}(E)=M(E)$ for each $\nu$-measurable $E$. If $f$ is a non-negative measurable function such that $\left\{\int_{E} f d M_{t}\right\}$ is a uniformly absolutely continuous class of totally finite measures, then for every set $E$ of finite $\nu$-measure, $\int_{E} f d M$ exists and

$$
\lim _{t \rightarrow \infty} \int_{E} f d M_{t}=\int_{E} f d M .
$$

Lemma (B). Let $\nu$ be a total measure. Let $\delta$ be a finite, finitely additive set function defined on the $\nu$-measurable sets, such that if $F$ is $\nu$-measurable and $\nu(F)<\infty$, then $\delta$ is an absolutely continuous measure on the $\sigma$-ring of $\nu$-measurable subsets of $F$. If there exists a nondecreasing sequence $\left\{F_{n}\right\}$ of $\nu$-measurable sets of finite $\nu$ such that $\bigcup_{n} F_{n}=\Omega=$ the entire space, and such that $\lim _{n \rightarrow \infty} \delta\left(F_{n}\right)$ $=\delta(\Omega)$, then $\delta$ is an absolutely continuous measure on the $\nu$-measurable sets.

Definition. Let $E$ be a $V$-measurable subset of $\Omega$. For each $t$, we define

$$
\mu_{t}(E, G)=V\left(E_{t} \cap G\right)
$$

for all $V$-measurable $G \subset \Omega$. If they exist, we define

$$
\mu_{-\infty}(E, G)=\lim _{t \rightarrow \infty} \mu_{-t}(E, G) \text {, and } \mu_{\infty}(E, G)=\lim _{t \rightarrow \infty} \mu_{t}(E, G) \text {. }
$$

REMARK (1). If $V(E)<\infty$, then $\mu_{t}(E, G)$ is the uniformly absolutely continuous conservative class of totally finite measures generated by $I(G)$ $=V(E \cap G)$.

Proof.Using Remarks (3) and (5) of $\$ 2$, we have,

$$
\mu_{t}(E, G)=V\left(E_{t} \cap G\right)=V\left(E \cap G_{-t}\right)=I\left(G_{-t}\right) .
$$

REMARK (2). $\mu_{-\infty}(E, G)$ exists for all measurable $E$ of finite $V$-measure and all measurable $G$ if and only if $\mu_{\infty}(E, G)$ does.

Proof. We use the fact that $V(G)=V\left(G^{*}\right)$ for all measurable $G$, and that $(F \cap G)^{*}=\left(F^{*}\right) \cap\left(G^{*}\right)$. By Condition (E), \$2, $V\left(\left(E^{*}\right)_{t} \Delta\left(E_{-t}\right)^{*}\right)=0$. Therefore, $\mu_{-t}(E, G)=V\left(E_{-t} \cap G\right)=V\left(\left(E_{-t}\right) * \cap G^{*}\right)=V\left(\left(E^{*}\right)_{t} \cap G^{*}\right)=\mu_{t}\left(E^{*}, G^{*}\right)$. This proves the remark.

THEOREM I. For each absolutely continuous $\sigma$-finite measure $I$, let $\left\{I_{t}\right\}$ be the conservative class of measures generated by $I$. The function $\mu_{\infty}(E, G)$ exists for each $E$ of finite measure if and only if $I_{\infty} \equiv \lim _{t \rightarrow \infty} I_{t}$ is, for arbitrary totally finite absolutely continuous measure $I$, an absolutely continuous totally finite measure on the $V$-measurable subsets of $\Omega$.

Proof. Assume that $\mu_{\infty}(E, G)$ exists. Then by Remark (2) above, so does $\mu_{-\infty}(E, G)$. Let $E \subset \Omega$ be $V$-measurable. We have by Remark (10) $\S 2, I_{t}(E)$ $=\int_{E_{-t}} \rho d V$, for some measurable $\rho \geqq 0$. The function $\rho$ is integrable by total 
finiteness of $I$. By Remark (11) $\left\{I_{t}\right\}$ is a uniformally absolutely continuous class of totally finite measures. Let $\mu_{-t}(E, G)$ be regarded as a measure of $G$, which we denote by $\mu_{-t}(G)$. We then have, $I_{t}(E)=\int_{\Omega} \rho d \mu_{-t}$. We consider two cases:

(i) $E \subset F_{n}$ for some integer $n$, where $F_{n}$ are the sets of condition (D) of $\$ 2$.

(ii) $E$ is not contained in $F_{n}$ for any $n$.

CASE (i). $E \subset F_{n}$ implies $E_{t} \subset\left(F_{n}\right)_{t}$ and that $V(E)<\infty$. Therefore $\mu_{t}(G)=0$ for all $V$-measurable subsets $G$ of $\Omega-\left(F_{n}\right)_{t}$. Therefore

$$
I_{t}(E)=\int_{\left(P_{n}\right)_{-t}} \rho d \mu_{-t}=\int_{P_{n}} \rho d \mu_{-t} .
$$

Since, by Remark (1) above, $\mu_{t}$ is a uniformly absolutely continuous class of totally finite measures, and since $\mu_{t}(\Omega) \leqq V\left(F_{n}\right)<\infty, \mu_{\infty}$ is a totally finite measure over the $V$-measurable sets, and so $\lim _{t \rightarrow \infty} I_{t}(E)$ exists by Lemma $A$.

CASE (ii). $E=U_{n} E \cap F_{n}$. We know, by Case (i) that $\lim _{t \rightarrow \infty} I_{t}\left(E \cap F_{n}\right)$ exists for each $n$. Further, because $I_{t}$ is totally finite, $\lim _{n \rightarrow \infty} I_{t}\left(E \cap F_{n}\right)$ also exists. Let $n \geqq m$. Then

$$
\begin{aligned}
\left|I_{t}\left(E \cap F_{n}\right)-I_{t}\left(E \cap F_{m}\right)\right| & =I_{t}\left(E \cap\left(F_{n}-F_{m}\right)\right) \leqq I_{t}\left(F_{n}-F_{m}\right) \\
& =\left|I_{t}\left(F_{n}\right)-I_{t}\left(F_{m}\right)\right|=\left|I\left(F_{n}\right)-I\left(F_{m}\right)\right| .
\end{aligned}
$$

But, by total finiteness of $I, I\left(F_{n}\right)$ converges, so that $I_{t}\left(E \cap F_{n}\right)$ converges uniformly in $n$ for all $t$. Therefore $\lim _{t \rightarrow \infty} I_{t}(E)$ exists and we can conclude that

$$
\begin{aligned}
I_{\infty}(E) & =\lim _{t \rightarrow \infty} I_{t}(E)=\lim _{t \rightarrow \infty} \lim _{n \rightarrow \infty} I_{t}\left(E \cap F_{n}\right)=\lim _{n \rightarrow \infty} \lim _{t \rightarrow \infty} I_{t}\left(E \cap F_{n}\right) \\
& =\lim _{n \rightarrow \infty} I_{\infty}\left(E \cap F_{n}\right) .
\end{aligned}
$$

Letting $E=\Omega$, this gives $\lim _{n \rightarrow \infty} I_{\infty}\left(F_{n}\right)=I_{\infty}(\Omega)$. Now $I_{\infty}$ is finitely additive and totally finite. Further, by uniform absolute continuity of $I_{t}, I_{\infty}$ is an absolutely continuous measure over $F_{n}$ for each $n$. Since $U_{n} F_{n}=\Omega$, Lemma B applies and $I_{\infty}$ is an absolutely continuous totally finite measure on the $V$ measurable subsets of $\Omega$. The rest of the proof of the theorem follows directly from Remark (1) above.

Whether or not $\mu_{\infty}(E, G)$ exists in the sense of Theorem I for the majority of systems considered in mechanics is a question which the author has so far been unable to answer. It appears to be true for nearly all separable systems. There are, however, exceptions, one of which is the harmonic oscillator. Separable systems for which $\mu_{\infty}$ does not always exist in some appropriately chosen $\Omega$ do seem, as Gibbs indicates, to be very exceptional. The existence of $\mu_{\infty}$ can be proved, for example, in the case of the rigid rotator and in the case of the ideal gas in a rectangular box. 
We now restrict the class of systems considered in this paper by one more condition on $f_{t}$.

Condition (F). $\mu_{\infty}(E, G)$ exists for each measurable $E$ of finite measure and each measurable $G$, where $E$ and $G$ are contained in $\Omega$.

From Theorem I it is now possible to conclude, by the Radon-Nikodym theorem that there exists a non-negative integrable function $\rho_{\infty}(X)$ for which

$$
I_{\infty}(E)=\int_{E} \rho_{\infty} d V
$$

We call $I_{\infty}(E)$ the equilibrium measure, and $\rho_{\infty}(X)$ an equilibrium density. It is easily seen that two equilibrium densities corresponding to a given $I$ are almost everywhere identical.

REMARK (3). $I_{\infty}\left(E_{s}\right)=I_{\infty}(E)$ for all real $s$ and measurable $E \subset \Omega$.

Interpreting Remark (3) in terms of $\rho_{\infty}$, we have, for $s \geqq 0$,

$$
\int_{E} \rho_{\infty}(X) d V=\int_{E_{s}} \rho_{\infty}(X) d V=\int_{E} \rho_{\infty}\left(f_{\bullet}(X)\right) d V
$$

so that $\rho_{\infty}(X)=\rho_{\infty}\left(f_{s}(X)\right)$ almost everywhere for $s \geqq 0$. In this sense, $\rho_{\infty}(X)$ is almost a constant of motion. This is as close as we can come within the scope of this paper to proving that $\rho_{\infty}$ satisfies Liouville's equation for equilibrium.

The next theorem gives information about the way in which certain ensemble averages at equilibrium are related to the corresponding quantities calculated for finite values of $t$. For a discussion of this aspect in special cases see a paper by $H$. Frisch [4]. The author feels that this theorem can be greatly improved upon, but that in order to do so, one must use more of the information contained in the equations of mechanics than is contained in Conditions (A)-(F) of this paper.

TheOREM II. Let $g$ be a measurable, non-negative function defined over $\Omega$. Let $I$ be a totally finite absolutely continuous measure over $\Omega$. If there exists a measurable function $\phi(X)$ defined over $\Omega$ with the following properties:

(i) $\phi \geqq g$ almost everywhere on $\Omega$;

(ii) $V(\{X: \alpha \leqq \phi(X)<\beta\})<\infty$ for each positive $\alpha$ and $\beta$;

and

(iii) $\phi\left(f_{t}(X)\right)=\phi(X)$ for all $X \in \Omega$, and $t \geqq 0$;

(iv) $\int_{\Omega} \phi d I$ exists;

then $\int_{\mathrm{a} g d I_{t}}$ and $\int_{\mathrm{a}} g d I_{\infty}$ exist and

$$
\lim _{t \rightarrow \infty} \int_{\mathbf{\Omega}} g d I_{t}=\int_{\mathbf{\Omega}} g d I_{\infty},
$$

where $I_{t}$ is the conservative measure generated by $I$. 
Proof. By Remark (11) $\S 2$ and Theorem $\mathrm{I},\left\{I_{t}\right\}$ is a uniformly absolutely continuous class of finite measures with $\lim _{t \rightarrow \infty} I_{t}=I_{\infty}$, where $I_{\infty}$ is a totally finite measure on the $V$-measurable subsets of $\Omega$. Let $\rho$ be the RadonNikodym derivative of $I$. Then since $\phi\left(f_{t}(X)\right)=\phi(X)$,

$$
\int_{E} g d I_{t} \leqq \int_{E} \phi d I_{t}=\int_{E_{-t}} \phi d I=\int_{E_{-t}} \phi \rho d V \leqq \int_{\Omega} \phi d I .
$$

Therefore, by Condition (B), and the integrability of $\phi$ with respect to $I$, $\left\{\int_{E} g d I_{t}\right\}$ is a uniformly absolutely continuous class of finite measures for $t \geqq 0$. Therefore, if $G$ is a set of finite $V$-measure, $\int_{G} g d I_{\infty}$ exists and

$$
\lim _{t \rightarrow \infty} \int_{G} g d I_{t}=\int_{G} g d I_{\infty}
$$

by Lemma A above. Let $G_{i}=\{X: 1 / i \leqq \phi(X)<i\}$ for positive integral $i$. By hypothesis $V\left(G_{i}\right)<\infty$. Therefore the limits

$$
\begin{aligned}
& \lim _{t \rightarrow \infty} \int_{G_{i}} g d I_{t}=\int_{G_{i}} g d I_{\infty}, \\
& \lim _{i \rightarrow \infty} \int_{G_{i}} g d I_{t} \leqq \int_{\mathbf{a}} g d I_{t}
\end{aligned}
$$

exist. Since $\phi\left(f_{t}(X)\right)=\phi(X),\left(G_{i}\right)_{-t}=G_{i}$ and therefore, if $i \geqq k$,

$$
\begin{array}{r}
\left|\int_{G_{i}} g d I_{t}-\int_{G_{k}} g d I_{t}\right|=\left|\int_{G_{i}-G_{k}} g d I_{t}\right| \leqq\left|\int_{G_{i}-G_{k}} \phi d I_{t}\right| \\
=\left|\int_{G_{i}} \phi d I_{t}-\int_{G_{k}} \phi d I_{t}\right|=\left|\int_{G_{i}} \phi d I-\int_{G_{k}} \phi d I\right| .
\end{array}
$$

Since $\left\{\int_{G_{i}} \phi d I\right\}$ converges for $i \rightarrow \infty,\left\{\int_{G_{i} g} g I_{i}\right\}$ converges uniformly for all $t$. Therefore,

$$
\lim _{i \rightarrow \infty} \int_{G_{i}} g d I_{\infty}
$$

exists and

$$
\int_{\Omega} g d I_{\infty}=\lim _{i \rightarrow \infty} \lim _{t \rightarrow \infty} \int_{G_{i}} g d I_{t}=\lim _{t \rightarrow \infty} \lim _{i \rightarrow \infty} \int_{G_{i}} g d I_{t} .
$$

Since $0 \leqq g \leqq \phi, g=0$ on $\Omega-U_{n} G_{n}$, and therefore

$$
\lim _{i \rightarrow \infty} \int_{G_{i}} g d I_{t}=\int_{\Omega} g d I_{t} \quad \text { for }|t| \leqq \infty .
$$

This proves the theorem. 
Theorem II would be more satisfactory without the requiremęnt that $\phi$ exist for a given $g$. If such an extended theorem is false, counter examples within the domain of classical mechanics are very difficult to find and are probably not simple. The author has yet to find any, and has, furthermore, actually proved the extended theorem for the rigid rotator. No discussion of this rather special case will be given here, except the remark that Theorem II above does not seem to be a very helpful stepping stone to the extended form.

In order to prove the next theorem, we note an inequality whose proof depends only on the simplest properties of convex sets. This inequality is found in Inequalities by Hardy, Littlewood and Polya. For another application of it, see also [6]. Let $\varphi(x)$ be a real convex function of a real variable $x$. Given any finite sets $\left\{x_{i}\right\}$ of real values of $x$ from the domain of $\varphi$, and $\left\{\lambda_{i}\right\}$ of positive real numbers,

$$
\varphi\left(\sum_{i} \frac{\lambda_{i}}{\lambda} x_{i}\right) \leqq \sum_{i} \frac{\lambda_{i}}{\lambda} \varphi\left(x_{i}\right),
$$

where $\lambda=\sum_{i} \lambda_{i}$. Note that $x \log x$ is convex for $x>0$. Let $E$ be a $V$-measurable set of finite measure. If $\rho$ is integrable over $E$ and if $\rho(X) \log \rho(X)$ is also, then it is easy to prove from the above inequality, an analogous one for integrals.

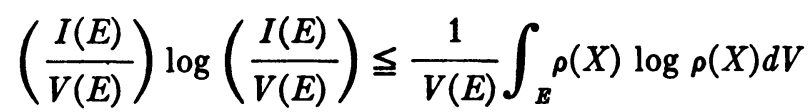

where $I(E)=\int_{E} \rho(X) d V$.

Let $I$ be an absolutely continuous totally finite measure on the $V$-measurable subsets of $\Omega$. We have, by Remark (10) $\$ 2$, an integrable function $\rho_{0}$ such that $I(E)=\int_{E} \rho_{0} d V$. By definition of $\rho_{\infty}$ we also have $I_{\infty}(E)=\int_{E} \rho_{\infty} d V$.

TheOREM III. Let $\int_{\Omega \rho_{0}} \log \rho_{0} d V$ exist. Then the integral $\int_{\Omega \rho_{\infty}} \log \rho_{\infty} d V$ exists in the extended sense and

$$
\int_{\Omega} \rho_{\infty} \log \rho_{\infty} d V \leqq \int_{\Omega} \rho_{0} \log \rho_{0} d V .
$$

Proof. It is convenient to define

$$
\begin{aligned}
S_{0}(E) & =\int_{E} \rho_{0} \log \rho_{0} d V, \\
S_{\infty}(E) & =\int_{E} \rho_{\infty} \log \rho_{\infty} d V .
\end{aligned}
$$

We wish to show that $S_{\infty}(E)$ is an extended valued set function and that 
$S_{\infty}(\Omega) \leqq S_{0}(\Omega)$. From inequality (1) above, we have

(2) $\left(\frac{I_{t}(E)}{V(E)}\right) \log \left(\frac{I_{t}(E)}{V(E)}\right)=\left(\frac{I\left(E_{-t}\right)}{V\left(E_{-t}\right)}\right) \log \left(\frac{I\left(E_{-t}\right)}{V\left(E_{-t}\right)}\right) \leqq \frac{S_{0}\left(E_{-t}\right)}{V\left(E_{-t}\right)}=\frac{S_{0}\left(E_{-t}\right)}{V(E)}$.

Let $i, j, k$, and $n$ be positive integers. Let $E_{k j}=\left\{X: 1 / j \leqq \rho_{\infty}(X)<k / j\right\}$ and let, for each $j$ and $k$,

$$
E_{i}^{n}=\left\{X: i / n j \leqq \rho_{\infty}(X)<(i+1) / n j\right\}
$$

Let $\chi_{j}^{n}$ be the characteristic function of $E_{i}^{n}$. Define fhe simple functions

$$
g_{k}^{n}(X ; t)=\sum_{i=n}^{n k-1} \chi_{i}^{n}(X) \lambda_{i}^{n}(t)
$$

where $\lambda_{i}^{n}(t)$ is defined by

$$
\lambda_{i}^{n}(t)=\left\{\begin{array}{cc}
\left(\frac{I_{t}\left(E_{i}^{n}\right)}{V\left(E_{i}^{n}\right)}\right) \log \left(\frac{I_{t}\left(E_{i}^{n}\right)}{V\left(E_{i}^{n}\right)}\right) & \text { when } V\left(E_{i}^{n}\right)>0, \\
0 & \text { when } V\left(E_{i}^{n}\right)=0 .
\end{array}\right.
$$

From inequality (2), we have $\lambda_{i}^{n}(t) \leqq S_{0}\left(\left(E_{i}^{n}\right)_{-t}\right) / V\left(E_{i}^{n}\right)$. It is an easy matter to verify that, for almost all $X \in E_{k}$,

$$
\lim _{n \rightarrow \infty} \lim _{t \rightarrow \infty} g_{k}^{n}(X ; t)=\rho_{\infty}(X) \log \rho_{\infty}(X) .
$$

Since, by integrability of $\rho_{\infty}(X), E_{k j}$ is of finite measure, Lebesgue's bounded convergence theorem gives

$$
\lim _{n \rightarrow \infty} \int_{E_{k j}} \lim _{t \rightarrow \infty} g_{k}^{n}(X ; t) d V=S_{\infty}\left(E_{k j}\right)
$$

By Remark (3), $\S 3, \rho_{\infty}\left(f_{t}(X)\right)=\rho_{\infty}(X)$ almost everywhere in $\Omega$ for all $t \geqq 0$. Therefore $V\left(E_{k j} \Delta\left(E_{k j}\right)_{-t}\right)=0$. Therefore $S_{0}\left(\left(E_{k j}\right)_{-\imath}\right)=S_{0}\left(E_{k j}\right)$.

Since $E_{k j}$ can be otherwise written as

$$
E_{k j}=\bigcup_{i=n}^{n k-1} E_{i}^{n}
$$

we have

$$
\left(E_{k j}\right)_{-t}=\bigcup_{i=n}^{n k-1}\left(E_{i}^{n}\right)_{-t}
$$

and because $E_{\imath}^{m} \cap E_{j}^{n}$ is empty for $i \neq j$, the definition of $S_{0}(E)$ gives 


$$
\sum_{i=n}^{n k-1} S_{0}\left(\left(E_{i}^{n}\right)_{-t}\right)=S_{0}\left(\left(E_{k j}\right)_{-\imath}\right)=S_{0}\left(E_{k j}\right)
$$

We now have,

$$
\begin{aligned}
S_{\infty}\left(E_{k j}\right) & =\lim _{n \rightarrow \infty} \int_{B_{k j}} \lim _{t \rightarrow \infty} g_{k}^{n}(X ; t) d V \\
& =\lim _{n \rightarrow \infty} \lim _{t \rightarrow \infty} \sum_{i=n}^{n k-1} V\left(E_{i}^{n}\right) \lambda_{i}^{n}(t) \\
& \leqq \lim _{n \rightarrow \infty} \lim _{t \rightarrow \infty} \sum_{i=n}^{n k-1} S_{0}\left(\left(E_{i}^{n}\right)_{-t}\right) \\
& =\lim _{n \rightarrow \infty} \lim _{t \rightarrow \infty} S_{0}\left(E_{k j}\right)=S_{0}\left(E_{k j}\right) .
\end{aligned}
$$

Now, since $\lim _{k \rightarrow \infty} S_{0}\left(E_{k j}\right)$ exists by hypothesis and since $S_{\infty}\left(E_{k j}\right)$ is a nondecreasing function of $k$ for $k>j$, the above inequality gives

$$
\lim _{k \rightarrow \infty} S_{\infty}\left(E_{k j}\right) \leqq \lim _{k \rightarrow \infty} S_{0}\left(E_{k j}\right) \text {. }
$$

Since $U_{k-1}^{\infty} E_{k 1}$ is the set on which $\rho_{\infty}(X) \log \rho_{\infty}(X) \geqq 0$, this proves that $S_{\infty}(G)$ is bounded from above. Therefore $S_{\infty}(G)$ is an extended valued set function. Let $E_{j}=\left\{X: \rho_{\infty}(X) \geqq 1 / j\right\}$. Then the preceding inequality gives

$$
S_{\infty}\left(E_{j}\right) \leqq S_{0}\left(E_{j}\right) \text {. }
$$

The theorem follows by letting $j \rightarrow \infty$.

By referring back to the case of the rigid rotator, it is easily seen that there are cases where strict inequality holds.

REMARK. Since $x \log x$ is bounded from below, the proof of Theorem III and Condition (D), $\$ 2$, imply that if $\rho_{0}=0$ almost everywhere in $\Omega-F_{n}$ for some $n, S_{\infty}(\Omega)$ exists as a finite number if $S_{0}(\Omega)$ does.

There is no possibility of proving the existence of $S_{\infty}(\Omega)$ as a finite number. A counter example can be given. Consider the case of the rigid rotator. We define the initial measure by means of the density function $\rho_{0}$ in the following way. Let

$$
g(p)=\frac{1}{p(\log p)^{3 / 2}}
$$

Then $\rho_{0}(X)$ is given by

$$
\rho_{0}(X)=\left\{\begin{array}{ccc}
0 & \text { when } p \leqq e \text { and }|q| \leqq \pi, \\
0 & \text { when } p>e \text { and }-\pi \leqq q<\pi-g(p), \\
1 / 2 & \text { when } p>e \text { and } 1>q \geqq \pi-g(p) .
\end{array}\right.
$$


It is easily seen by direct calculation that $\int_{\Omega} \rho_{0}(X) d V=1$, and $S_{0}(\Omega)=-\log 2$. The equilibrium density, however, is almost everywhere equal to

$$
\rho_{\infty}(X)=\left\{\begin{array}{cc}
g(p) / 4 \pi & \text { when } p>e, \\
0 & \text { when } p \leqq e
\end{array}\right.
$$

for which $S_{\infty}(\Omega)$ can be found by direct calculation to be negatively infinite.

\section{REFERENCES} ter XII.

1. J. W. Gibbs, Elementary principles of statistical mechanics, Scribner's Sons, 1902, Chap-

2. R. C. Tolman, The principles of statistical mechanics, Oxford University Press, 1938, \$51, Chapter VII.

3. H. Grad, Principles of the Kinetic Theory of Gases, Handbuch der Physik, Band XII, 1959.

4. H. L. Firsch, An approach to equilibrium, Phys. Rev. vol. 109 (1958) pp. 22-29.

5. P. R. Halmos, Measure theory, Van Nostrand, 1950.

6. A. I. Khinchin, Mathematical foundations of information theory, Dover, 1957, p. 6.

7. N. Steenrod, The topology of fibre bundles, Princeton, Princeton University Press, 1951, Part I, 81.8.

Göttingen, Germany 